\title{
Numérique et accessibilité dans l'éducation et en formation
} Présentation du dossier

\author{
Hervé BENOIT \\ INS HEA - Université Paris Lumières (UPL) \\ EA 7287 - Grhapes \\ Teresa ASSUDE \\ Aix-Marseille Université (AMU) \\ EA 4671 - ADEF \\ Jean-Michel PEREZ \\ Université de Lorraine \\ EA 2310 - LISEC
}

\section{$\mathbf{U}$}

$\mathrm{N}$ certain nombre de travaux ont mis en évidence le risque que la fracture numérique accentue la fracture sociale. Dans cette perspective, on peut être amené à se demander comment donner à l'école les moyens de prévenir ce risque en favorisant l'accès au numérique pour tous les élèves. Mais le développement actuel du numérique dans la vie quotidienne et dans les pratiques professionnelles peut aussi être envisagé sous un autre angle, non plus comme obstacle à l'acquisition des savoirs, mais comme levier de l'accessibilité didactique et pédagogique pour tous les degrés d'enseignement, du primaire à l'université.

$C^{\prime}$ est ainsi que ce dossier, issu des communications présentées au colloque Ophris des 7 et 8 juin 2016 à I'INS HEA, veut interroger le rôle du numérique en tant que levier pour la construction d'une école inclusive et potentiel incubateur de nouvelles pratiques éducatives et pédagogiques dans l'enseignement scolaire et dans le cadre de la formation des futurs enseignants. Nos questions sont alors: En quoi le numérique (en supposant qu'il soit accessible) est-il l'un des moyens de construction d'une école inclusive? Sur quels types d'usages du numérique peut-on s'appuyer pour développer l'accessibilité de tous aux savoirs qui est la condition première d'un système éducatif inclusif?

C'est autour de deux axes de recherche que s'organise le présent dossier: d'une part, celui des usages, dans une perspective inclusive, des technologies numériques dans les degrés l'enseignement scolaire, mais aussi dans un cadre éducatif plus large où les usages qu'en font les jeunes peuvent devenir erratiques, et, d'autre part, 
celui de la contribution de ces outils à la formation des enseignants aux pratiques professionnelles de l'éducation inclusive, afin de leur permettre d'acquérir les compétences nécessaires pour comprendre la diversité des besoins et mettre en œuvre les moyens d'y répondre.

Dans le premier axe, ce sont des usages du numérique qui seront au cœur des contributions afin de montrer en quoi et comment ils peuvent faciliter, en général, l'accès de l'ensemble des élèves aux savoirs et savoir-faire scolaires et, en particulier, réduire les obstacles aux apprentissages que rencontrent ceux qui sont en situation de handicap ou de difficulté. L'enjeu est de poser les jalons pour constituer une culture de l'éducation pour tous et de la pédagogie numérique inclusive qui soit opérante dès l'école et qui traverse ensuite le champ des études secondaires, universitaires et de la formation professionnelle. La question est alors de savoir de quels outils numériques se saisir, quels usages promouvoir et pour quels gains d'accessibilité? Le deuxième axe concerne l'apport particulier des ressources numériques, et notamment celles de la vidéo, à la formation des enseignants aux pratiques professionnelles de l'éducation inclusive. Or la construction des compétences dans ce domaine est tout autant subordonnée à la maîtrise d'outils et de technologies numériques génériques et spécifiques qu'à celle de l'ingénierie didactique qui permet de les faire contribuer à cet objectif. L'un des questionnements exploré par ce dossier est alors le suivant: en quoi le numérique contribue-t-il au travail de formation, et en quoi le questionne-t-il dans le même mouvement?

Les deux premiers articles s'intéressent aux conditions dans lesquelles les ressources numériques peuvent favoriser l'accessibilité aux apprentissages et aux savoirs. Dans le premier, Teresa Assude, se fondant sur un travail de recherche relatif à l'intégration des calculatrices à l'école primaire, aborde l'usage des outils numériques sous l'angle de la plus-value didactique susceptible d'en résulter. II apparaît que celle-ci se présente comme une fonction à six variables, identifiées dans les discours et les pratiques: la valeur symbolique et sémiotique, la valeur instrumentale, la valeur praxéologique, la valeur d'étude, la valeur d'usage et les résistances. L'hypothèse de travail est alors que l'intégration des outils numériques dans l'action didactique des enseignants est subordonnée au niveau plus ou moins élevé de cette plus-value. Ainsi le numérique peut-il contribuer à l'accessibilité didactique, sous réserve toutefois que des difficultés instrumentales ne viennent pas faire obstacle à l'accès aux savoirs, que l'usage de l'outil n'altère pas l'enjeu d'apprentissage et qu'il s'inscrive dans le système de ressources constituant le milieu de la situation. C'est donc en lien avec la situation d'enseignement, les enjeux de savoir, les besoins des élèves, les fonctions de l'outil et la plus-value qu'il apporte dans l'économie de l'apprentissage, que doit être pensé l'apport du numérique. De leur côté, Hervé Benoit et Sylviane Feuilladieu s'attachent à définir, dans le champ des Environnements informatiques pour l'apprentissage humain (EIAH), ce qu'ils nomment l'opérationnalité inclusive des ressources numériques, c'est-à-dire leur capacité à concourir à l'accessibilité pédagogique universelle. Partant du constat que, dans le domaine du handicap et de la difficulté scolaire, les ressources numériques se sont historiquement développées sous la forme d'aides techniques personnalisées en relation avec 
des déficits et/ou des troubles moteurs, cognitifs ou sensoriels, ils interrogent les potentialités inclusives du numérique, au-delà de l'usage compensatoire qui en est fait couramment, et montrent qu'elles peuvent être situées et caractérisées au moyen d'un repère constitué par le croisement de deux axes: le premier est celui du degré de didactisation; le second relie le pôle individuel de la compensation au pôle collectif de l'accessibilité. La carte heuristique ainsi constituée permet de faire apparaître un troisième axe, le vecteur dynamique de l'opérationnalité inclusive, qui correspond à l'effectivité du processus d'accessibilisation pédagogique et didactique.

Suivent cinq articles qui montrent comment, dans différents contextes d'enseignement scolaire, des ressources numériques peuvent produire des gains d'accessibilité pédagogique pour des élèves présentant des troubles d'ordre moteur, cognitif et sensoriel. C'est tout d'abord Édith Petitfour qui cherche à mieux comprendre la nature des difficultés des élèves dyspraxiques accueillis en milieu ordinaire au cycle 3 dans des tâches de construction géométrique. Considérant, en appui sur des résultats de recherche, qu'il serait contre-productif de tenter de restaurer par la rééducation les habiletés motrices et les compétences organisationnelles de ces élèves dans l'espoir qu'ils acquièrent, à terme, des connaissances géométriques, l'auteur montre que l'utilisation d'un logiciel de géométrie dynamique peut permettre un gain substantiel d'accessibilité à ces connaissances et contribue à rendre capacitantes les conditions de l'apprentissage. Ainsi, par exemple, est-il possible de remplacer, pour l'élève dyspraxique, la réalisation d'actions instrumentées, qui accompagne classiquement l'apprentissage géométrique au cycle 3, par une pratique langagière qui se substitue à l'action et permet ainsi d'alléger le coût lié aux efforts manipulatoires. Hélène Terrat et Jack Sagot étudient quant à eux l'intérêt et les perspectives d'accessibilité pédagogique ouvertes par l'utilisation d'un outil numérique traçant dédié à l'apprentissage pour des élèves présentant des troubles praxiques, graphomoteurs et mnésiques. L'outil informatique rend l'écriture accessible à ces élèves et les difficultés de lecture sont contournées par le retour vocal. Mais au-delà d'une suppléance aux troubles, le retour différé et la réflexivité augmentée immédiate supportée par le traceur soutiennent la pensée procédurale et la planification de la tâche, favorisent l'apprentissage autonome et renforcent le sentiment d'auto-efficacité. Les deux articles suivants, celui de Cendrine Mercier, Patrice Bourdon et Gaëlle Lefer d'abord, puis celui de Philippe Garnier, concernent I'utilisation de tablettes tactiles en milieu scolaire auprès d'enfants avec Troubles du spectre de l'autisme (TSA) et analysent I'un et l'autre I'apport induit en terme d'accessibilité aux apprentissages relationnels et scolaires. Pour le premier, il s'agit de l'introduction dans trois contextes institutionnels différents (Ulis école, IME et Sessad), d'un support numérique nommé çATED. Cette application fonctionne comme un agenda numérique qui, en proposant un découpage de la journée, permet aux enfants de se repérer dans le temps et l'espace. L'étude sur une année montre que les enfants s'approprient progressivement l'outil et l'utilisent pour mieux communiquer entre pairs ou avec les professionnels, ce qui favorise l'entrée dans les activités d'apprentissage. Le second relate les premiers mois d'une expérimentation dans laquelle une cohorte composée de 23 élèves avec TSA de 
7 à 13 ans apprennent du vocabulaire et à reconnaître directement certains mots par l'intermédiaire d'une tablette numérique ainsi qu'avec du matériel papier. Si les résultats confirment un certain nombre de points mentionnés dans la littérature, ils apportent aussi des éléments originaux, comme par exemple le fait que les élèves avec TSA sont capables de réaliser un certain nombre d'activités sur la tablette qu'ils ne peuvent effectuer en situation non numérique. Enfin, pour clore cet ensemble d'articles, la contribution professionnelle de Didier Flory décrit les conditions dans lesquelles il a élaboré, grâce au soutien du ministère de l'Éducation nationale, un site éducatif et pédagogique inclusif, lire-ensemble.com, accessible aux élèves sourds communiquant en langue des signes, mais aussi à tous les élèves entendants et bientôt à des jeunes avec Troubles du spectre de l'autisme. Dans l'esprit de son concepteur, il ne s'agit en aucun cas d'un site spécialisé, mais d'un espace de rencontre avec la lecture (et avec d'autres activités culturelles et pédagogiques), destiné à tous, tout en étant accessible aux Sourds.

Entre l'ensemble constitué par les cinq articles précédents, centrés sur les gains d'accessibilité aux apprentissages dus au numérique, et les trois derniers qui sont consacrés à l'apport du numérique dans la formation des enseignants aux pratiques pédagogiques inclusives, deux textes font transition, qui élargissent la problématique des usages inclusifs du numérique à la dimension ludique, que celle-ci s'articule à l'enseignement à travers les jeux sérieux, ou qu'elle prenne des formes erratiques, lorsque l'accès illimité à Internet conduit les jeunes à certaines formes d'addictions. Pour Mathieu Muratet, auteur du premier de ces deux textes, les jeux sérieux sont aujourd'hui en plein développement et sont devenus plus accessibles, en particulier depuis le déploiement des tablettes numériques dans l'école, ce qui justifie de présenter cette ressource et d'en questionner la dimension pédagogique. Il apparaît en effet que si le jeu sérieux apporte un contexte scénaristique particulièrement attractif (déplacement dans un monde virtuel, accomplissement de quêtes annexes, configuration de son personnage) qui diffère du scénario pédagogique classique, le problème qui se pose à l'enseignant qui en fait usage est de disposer d'informations sur l'activité de l'apprenant et sur les compétences construites ou non au cours de l'activité ludique. Le problème de l'enseignant devient alors celui de l'informaticien, qui doit identifier les compétences à évaluer; établir les critères qui permettent de les évaluer; identifier les activités et les situations dans lesquelles il est possible observer l'activité de l'apprenant et obtenir les preuves permettant de la relier aux compétences. Pour Stéphane Blocquaux, qui est l'auteur du second texte, la disponibilité des enfants au numérique semble créer les conditions d'une certaine errance, qui rend notamment possible l'accès à des images à caractère pornographique. Deux recherches scientifiques conduites sur les thématiques de la cyberaddiction (ou netaholisme) et du cybersexe engagent selon lui à réfléchir aux diverses formes que pourrait prendre une véritable éducation au virtuel. Quelles que soient les mesures actuellement envisagées au niveau national (filtrage, voire d'interdiction totale des images à caractère sexuel), il semble en tout cas qu'elles risqueraient d'être inefficaces si elles n'étaient pas accompagnées d'outils pédagogiques portés par l'école (vidéo éducatives, sites web, témoignages d'acteurs, 
etc.) permettant aux parents de discuter de la question de la pornographie sur Internet au sein de leurs foyers, avec leurs enfants.

Les trois derniers articles, qui ferment ce dossier, interrogent l'apport du numérique, et notamment celui de la vidéo, dans la formation des enseignants aux pratiques pédagogiques inclusives. Le premier, celui d'Isabelle Nédélec-Trohel, repose sur l'étude exploratoire d'un dispositif de formation par alternance préparant à l'examen (Capa-SH) des maîtres chargés de l'aide à dominante pédagogique (maîtres E) enseignant à l'école primaire. II a pour objet de montrer en quoi et comment une Analyse de pratique réflexive (APR) en formation ASH permet de mettre au jour des obstacles à I'apprentissage en numération. Dans cette perspective, il est remarquable que la vidéo avec transcript fonctionne dans le dispositif APR comme outil didactique d'aide, nécessaire à la construction d'objets d'étude participant de la formation aux pratiques inclusives des professeurs spécialisés. Le second, auquel ont contribué Jean-Michel Perez, Teresa Assude, Géraldine Suau et Jeannette Tambone, s'intéresse également aux usages de la vidéo et à leurs effets sur les pratiques enseignantes inclusives. C'est dans le cadre d'un dispositif de recherche phénoméno-praxéologique, mis en place depuis 2012 sur les Pratiques inclusives en milieu scolaire ordinaire (PIMS), que s'inscrit cette problématique. L'originalité de ce dispositif consiste à articuler quatre types d'institutions, dans lesquelles les acteurs assument des topos différents: I'institution d'action en classe, l'institution d'analyse des praxéologies professionnelles (analyse d'un extrait vidéo d'une captation de séance), l'institution de production des praxéologies professionnelles et l'institution de transposition didactique en formation. L'article se focalise sur la quatrième institution, qui est une institution de formation. II fait apparaître que I'utilisation en formation, avec de futurs enseignants, des mêmes extraits vidéo qui avaient constitué le support de l'analyse des praxéologies professionnelles, produit une nouvelle dynamique qui permet d'éclairer des points restés aveugles, comme par exemple la question de la co-responsabilité de la dynamique inclusive. Enfin, c'est à deux professeurs des écoles maîtres formateurs, Nelly Carême et Hélène Smouts, toutes deux engagées depuis plus de trois ans dans le dispositif de recherche PIMS décrit dans I'article qui précède, que revient I'honneur de clore ce dossier. Ensemble, par un discours à deux voix, elles éclairent les raisons personnelles et professionnelles de leur participation à cette recherche, précisent en quoi cette implication a fait évoluer leurs pratiques, s'interrogent sur ce qui se joue dans une situation de classe, et, dans un temps conclusif, expliquent en quoi leur participation à ce dispositif questionne leur activité et leur rôle de formatrices auprès des étudiants fonctionnaires stagiaires de l'Espé. 